\section{P198 DOES GUM SPECIALITY TRAINING PREPARE NEW CONSULTANTS TO MANAGE SEXUAL DYSFUNCTION?}

${ }^{1}$ Jane Nicholls*, ${ }^{2}$ Pippa Green, ${ }^{3}$ Karl Hollows, ${ }^{4}$ David Goldmeier. ${ }^{1}$ Bristol Sexual Health Centre, Bristol, UK; ${ }^{2}$ University Hospital of South Manchester NHS FT, Manchester, UK; ${ }^{3}$ Cobridge Sexual Health Service, Staffordshire and Stoke on Trent NHS Partnership Trust, Stoke on Trent, UK; ${ }^{4}$ Jefferiss Wing, St. Mary's Hospital, London, UK

\subsection{6/sextrans-2015-052126.242}

Background/introduction Service provision for patients with sexual dysfunction (SD) in the UK varies according to locality and available expertise. Speciality training in SD may be variable and poorly standardised.

The 2010 GUM curriculum is due for review in 2015. The opinion of senior trainees and new consultants will help inform these curriculum developments.

Aim(s)/objectives We aim to establish

- whether new consultants feel adequately equipped to manage patients with SD

- what additional training is currently being undertaken

- whether additional training opportunities would be welcome

Methods An electronic survey was distributed to 51 trainees within 24 months of CCT and 19 new consultants.

Results The response rate was 39\% (27/70) from 9 deaneries. $92 \%(24 / 26)$ felt that having training in SD as a GUM physician was important $(46 \%)$ or very important $(46 \%)$. Most trainees had had some exposure to informal teaching $89 \%$ (24/27) or departmental teaching 63\% (17/27) but very few had formal training. Only $8 \%(2 / 26)$ of respondents felt their training had adequately equipped them to manage SD. 46\% (12/26) felt equipped to some extent but $31 \%(8 / 26)$ did not feel adequately equipped to manage SD. $88 \%$ (23/26) felt they would benefit from further training.

Discussion/conclusion Many senior trainees and new consultants do not feel equipped to manage SD. The ability to recognise and appropriately refer patients with SD is essential for any GUM clinician. The 2015 curriculum review will help standardise core training in SD, as well as providing opportunities for those who wish to deliver specialised services in future.

\section{P199 CHANGING TEENAGERS' PERSPECTIVES ON THEIR SEXUAL HEALTH: RESULTS FROM AN INNOVATIVE EDUCATIONAL PROGRAMME IN UK SECONDARY SCHOOLS}

${ }^{1}$ Miriam Hillyard*, ${ }^{2}$ Beatrice Cockbain. ${ }^{1}$ Imperial College Healthcare NHS Trust, London, UK; ${ }^{2}$ Royal Free London NHS Foundation Trust, London, UK

\subsection{6/sextrans-2015-052126.243}

Background/introduction UK schools are not obliged to provide comprehensive sex and relationships education (SRE). SRE is frequently outdated, taught by non-specialists, and covers only the technicalities of heterosexual sex and sexually-transmitted diseases.

Aim(s)/objectives We aimed to deliver a peer-led programme of age-appropriate sessions covering sexual, physical, and psychological health, inclusive of non-heterosexual and non-cisgender identities. Sessions were designed to empower young people aged 1118 to discuss these topics in a non-judgemental environment.

Methods 50-minute sessions encompassed body image, drugs and alcohol, sex and sexual risk taking, or contraception.
Trained university student volunteers employed games, small group discussions, quizzes, and visual media. Volunteer to pupil ratio averaged 1:8. Pupils were encouraged to ask questions and reflect throughout. Anonymous written feedback assessed pupils' enjoyment of the sessions, volunteers' teaching ability, and impact of the sessions on their self-perception.

Results 876 feedback forms were completed. 91.8\% of pupils enjoyed the sessions and $93.0 \%$ rated them as well taught. $61.9 \%$ of pupils reported the session to have changed the way they felt about themselves or their health. Free text comments from the remaining $38.1 \%$ indicated prior comfort with navigating health issues. Forms also showed high levels of satisfaction with the opportunity to receive non-judgemental, comprehensive responses from relatable peer-educators.

Discussion/conclusion Comprehensive SRE delivered by knowledgeable peer-educators allows teenagers to freely discuss issues surrounding their sexual and mental health, empowering them to make informed decisions and potentially affecting their risktaking behaviours. This programme demonstrates an innovative but easily replicable means of providing this education.

\section{P200 A FACILITY TO ENABLE HIGH-QUALITY, TIME-EFFICIENT EVALUATIONS OF DIAGNOSTICS FOR STIS}

${ }^{1}$ Emma Harding-Esch*, ${ }^{2}$ Marcus Pond, ${ }^{2}$ Achyuta Nori, ${ }^{2}$ Sebastian Fuller, ${ }^{2} S$-L Christine Chow, ${ }^{3}$ Rebecca Howell-Jones, ${ }^{2}$ Catherine Hall, ${ }^{2}$ Mark Harrison, ${ }^{1}$ Anthony Nardone, ${ }^{2}$ Tim Planche, ${ }^{2}$ Philip Butcher, ${ }^{1}$ Catherine Lowndes, ${ }^{2} S$ Tariq Sadiq. ${ }^{1}$ Public Health England, London, UK; ${ }^{2}$ St George's, University of London, London, UK; ${ }^{3}$ Oxford School of Public Health, Oxford, UK

\subsection{6/sextrans-2015-052126.244}

Background/introduction Control of STIs is challenged by inadequate access to prompt diagnosis and treatment for patients and partners. Novel point-of-care diagnostics have real potential to address some of these challenges but their robust evaluation, and hence utility, is hampered by the ethics and regulatory landscape that confronts industry and academia.

Aim(s)/objectives To develop a diagnostics and clinical facility to deliver high-quality, time-efficient diagnostic evaluations for STIs.

Methods A multi-institutional and disciplinary group $\left(e \mathrm{STI}^{2}\right)$ including clinical, public health and social scientists, microbiologists, clinicians, trial coordinators, and North American and European regulatory expertise was established. An 'overarching' ethics, favourable costing, and regulatory framework was carefully developed and put in place to enable any new diagnostic evaluation involving residual and/or additional-to-routine patient-consented samples to start promptly without requiring lengthy ethics applications. Strong working relationships with multiple GUM clinics were developed to overcome the potential for clinic fatigue, and Good Clinical Laboratory Practice Standard Operating Procedures were enabled.

Results Since February 2012, the network has conducted several evaluations with both academia and industry, spanning initial 'proof of concept' projects using residual samples, multi-site diagnostic evaluations involving $>800$ additional-to-routine patient samples completed in four months, and service evaluations of CE-marked assays. A diagnostic evaluation to support an application for regulatory approval will be taking place in 2015 . Discussion/conclusion The development of a diagnostic facility for STIs that fast-tracks high quality diagnostic evaluations is 